\title{
Intrinsic normalized emittance growth in laser-driven electron accelerators
}

\author{
M. Migliorati, ${ }^{1,2}$ A. Bacci, ${ }^{3}$ C. Benedetti,${ }^{4, *}$ E. Chiadroni,${ }^{3}$ M. Ferrario, ${ }^{3}$ A. Mostacci, ${ }^{1,2}$ \\ L. Palumbo, ${ }^{1,2}$ A. R. Rossi, ${ }^{3}$ L. Serafini, ${ }^{5}$ and P. Antici ${ }^{1,2,3, \dagger}$ \\ ${ }^{1}$ Sapienza, University of Rome, Dipartimento SBAI, Via A. Scarpa 14, 00161 Rome, Italy \\ ${ }^{2}$ Istituto Nazionale di Fisica Nucleare (INFN), Sezione di Roma, Dipartimento di Fisica, Sapienza, \\ University of Rome, Piazzale Aldo Moro 2, 00185 Rome, Italy \\ ${ }^{3}$ INFN, Laboratori Nazionali di Frascati, Via E. Fermi 40, 00044 Frascati, Italy \\ ${ }^{4}$ University of Bologna and INFN, Bologna, Italy \\ ${ }^{5}$ INFN-Milan and University of Milan, Department of Physics, Via Celoria 16, 20133 Milan, Italy
}

(Received 22 December 2011; published 10 January 2013)

\begin{abstract}
Laser-based electron sources are attracting strong interest from the conventional accelerator community due to their unique characteristics in terms of high initial energy, low emittance, and significant beam current. Extremely strong electric fields (up to hundreds of $\mathrm{GV} / \mathrm{m}$ ) generated in the plasma allow accelerating gradients much higher than in conventional accelerators and set the basis for achieving very high final energies in a compact space. Generating laser-driven high-energy electron beam lines therefore represents an attractive challenge for novel particle accelerators. In this paper we show that laser-driven electrons generated by the nowadays consolidated TW laser systems, when leaving the interaction region, are subject to a very strong, normalized emittance worsening which makes them quickly unusable for any beam transport. Furthermore, due to their intrinsic beam characteristics, controlling and capturing the full beam current can only be achieved improving the source parameters.
\end{abstract}

DOI: 10.1103/PhysRevSTAB.16.011302

PACS numbers: 41.75.Jv, 41.85.Ja

Conventional particle accelerators have been one of the most important scientific instruments in the past decades. Users of accelerators are constantly demanding increasing particle energy in order to perform upfront science [1]. Unfortunately, the accelerating field of conventional accelerators is limited by breakdown issues, which leads to maximum accelerating gradients currently slightly higher than $100 \mathrm{MeV} / \mathrm{m}$ (at a reasonable breakdown rate and using standing wave structure) [2]. Consequently, high energies can only be achieved with large accelerator facilities which nowadays are approaching the limit of what becomes bearable in terms of size and costs.

An alternative to conventional accelerators are laserdriven plasma accelerators ([3] and references therein), which can generate accelerating electric fields up to hundreds of $\mathrm{GV} / \mathrm{m}$, about a thousand times higher than what can be obtained in conventional accelerators, and very intense magnetic fields [4]. Concerning laser-generated electrons, currently, multihundred $\mathrm{MeV}$ electron beams have been experimentally obtained on typical 10-100 TW laser systems [5] with maximum electron energies reaching up to $1 \mathrm{GeV}$ [6]. These beams possess a number of outstanding properties, such as ultrashort pulse duration

\footnotetext{
*Now at LBNL, Berkeley, California 94720-8211, USA.

patrizio.antici@uniroma1.it
}

Published by the American Physical Society under the terms of the Creative Commons Attribution 3.0 License. Further distribution of this work must maintain attribution to the author(s) and the published article's title, journal citation, and DOI. (fs scale), high peak currents (kA range), and excellent emittance $(<1 \mathrm{~mm} \mathrm{mrad})$ at the plasma-vacuum interface. Substantial effort is currently undertaken to characterize these beams, trying to optimize their characteristics ([7-10] and references within). Despite these efforts, laser-generated electron beam lines have still not been obtained with reasonable beam parameters and using them as a replacement of conventional accelerators is currently extremely challenging [11].

In this paper, we analyze the evolution of the laserdriven beam source parameters for a successive capture and transport of the particles. We focus our study on particles obtained on current consolidated state-of-the-art laser facilities reaching the hundred-TW regime. In this respect, we have concentrated on electron parameters achieved using the self-injection laser wakefields acceleration scheme known as the 3D bubble regime $[12,13]$ although our conclusions are valid also for other experimental setups in the self-injection bubble regime. Simulations of the laser-plasma interaction have been performed using the 3D particle-in-cell (PIC) code ALADYN [14], optimizing the laser-plasma interaction in order to achieve highest electron energies [15]. The simulation considers a 200 TW laser [16] with wavelength $\lambda=$ $0.8 \mu \mathrm{m}$, main laser pulse to prepulse contrast ratio of $10^{10}$, pulse duration full-width-half-maximum (FWHM) $\tau=30 \mathrm{fs}$, and waist $=15.5 \mu \mathrm{m}$, delivering an intensity of $I=5 \times 10^{19} \mathrm{~W} / \mathrm{cm}^{2}$. For the interaction we have considered a plasma length of $4.1 \mathrm{~mm}$ with a plasma ramp of $100 \mu \mathrm{m}$ and electron density $3 \times 10^{18} \mathrm{~cm}^{-3}$ which generates electrons with a charge of $700 \mathrm{pC}$ and $910 \mathrm{MeV}$ 
average energy. The main parameters, in rms values, are energy spread $\sigma_{E}=6.4 \%$, bunch length $\sigma_{z}=2 \mu \mathrm{m}$, transverse beam size $\sigma_{x}=0.5 \mu \mathrm{m}$, transverse divergence $\sigma_{x^{\prime}}=$ $3 \mathrm{mrad}$, geometric emittance $\varepsilon=1.4 \times 10^{-3} \mathrm{mmmrad}$, and normalized emittance $\varepsilon_{n}=2.5 \mathrm{~mm}$ mrad. The initial energy distribution and current of the bunch are shown in Fig. 1. Albeit there are several publications reporting about the laser-driven electron parameters, we will concentrate here on values obtained by our simulations, since only these can shed light on parameters such as beam emittance or the behavior of each single particle. These values are consistent with typical experimental setups $[15,17]$.

In the above-mentioned acceleration regime, the impinging laser pulse displaces electrons from the initially neutral plasma, creating a bubble-shaped region of ions. These electrons tend to cumulate in a thin layer surrounding the bubble. The charge separation generates very intense electromagnetic fields (up to $\mathrm{TeV} / \mathrm{m}$ ). If the normalized laser parameter $a_{0}$ is sufficiently high (>2.5-3), the electrons with longitudinal velocity component greater than the bubble speed are eventually captured and accelerated by the strong longitudinal field. The resulting normalized mean particle energy (temperature), in $\mathrm{MeV}$ units, is in the order of $a_{0}$ (few MeVs). Despite the high
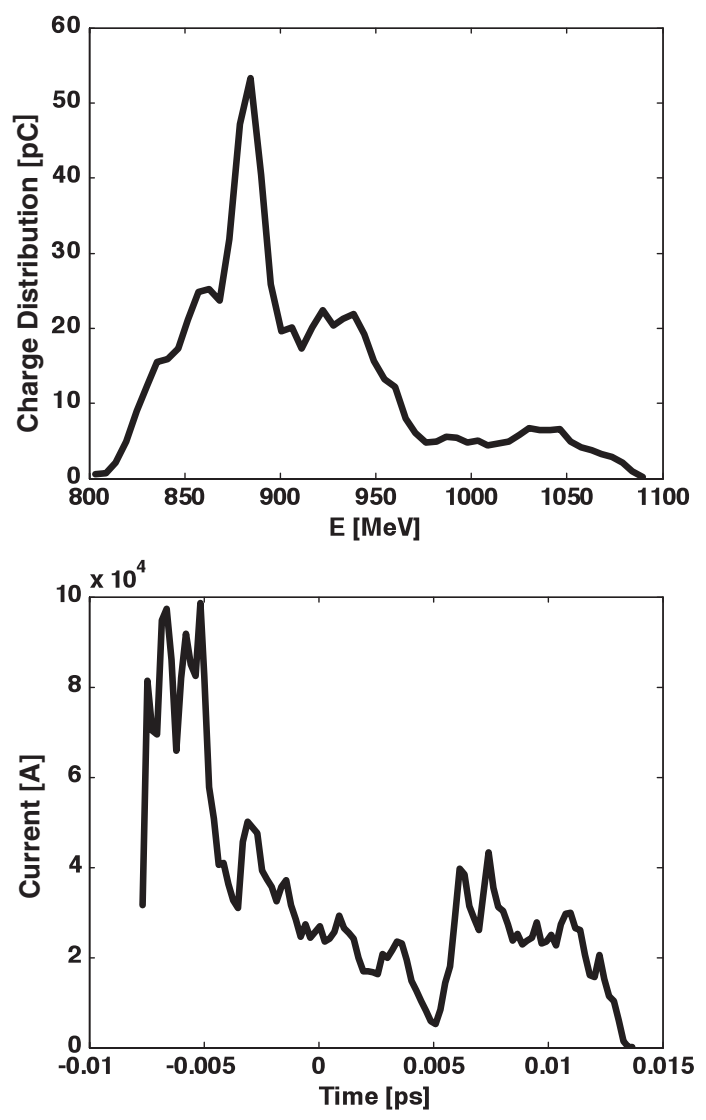

FIG. 1. Charge distribution vs energy (top) and current vs time (bottom) of the initial electron distribution as obtained with the PIC code ALADYN. transverse momenta, such particles form a well behaved beam, since the focusing components of the electromagnetic fields generated by the bubble are very intense and the space charge effects are partially compensated by the ions. The resulting bunch is emittance dominated even if its peak current can be as high as $100 \mathrm{kA}$.

In conventional radio frequency (rf) photoinjectors (i.e. a source of high quality electron beams), electrons are extracted from the photocathode with a random momentum of a few $\mathrm{eV} / \mathrm{c}$ and are then accelerated to few $\mathrm{MeV}$ energies. Assuming that during the longitudinal acceleration the transverse momentum of each particle remains constant, its divergence is reduced by a factor of $10^{6} \mathrm{after}$ acceleration. In comparison, for a plasma accelerated beam, reaching an energy in the order of the $\mathrm{GeV}$ in a very short distance, such a divergence reduction is only $10^{3}$ since the thermal momentum of the electrons within the plasma is in the order of a few $\mathrm{MeV} / \mathrm{c}$.

When exiting the laser-plasma region, particles move from an extremely intense focusing field, generated inside the bubble, to a free space where the focusing effect suddenly vanishes. A possible way to overcome the increasing divergence in the following free space is to apply, in the immediate proximity, strong magnetic fields by means of quadrupoles or solenoids.

However, from our beam parameters we obtain that the Twiss beta function, defined as $\beta_{x}=\sigma_{x}^{2} / \varepsilon$, is as low as a fraction of $\mu \mathrm{m}$, many orders of magnitude lower than what is generally found in conventional accelerators. The consequence is a very rapid increase of the transverse correlation (which initially is zero and, in our case, scales with $1 / \beta_{x_{0}}$, where $\beta_{x_{0}}$ is the initial value of $\beta_{x}$ ) in a drift. For example, in our case, we find an increase of 60 times in the transverse dimension after only $1 \mathrm{~cm}$ drift. Thus, unlike conventional accelerators, electrons undergo a normalized emittance increase during a drift. Such behavior can be understood by using the general definition of normalized emittance that reads

$$
\varepsilon_{n}^{2}=\left\langle x^{2}\right\rangle\left\langle\beta^{2} \gamma^{2} x^{\prime 2}\right\rangle-\left\langle x \beta \gamma x^{\prime}\right\rangle^{2}
$$

with $\beta=v / c$, where $v$ is the particle velocity and $c$ the speed of light, and $\gamma$ being the particle relativistic factor, $x$ and $x^{\prime}$ the transverse position and its divergence, and $\langle\gamma\rangle$ the average relativistic factor, and considering that the correlation between the energy and transverse position is negligible (as in a drift without collective effects), we find

$$
\varepsilon_{n}^{2}=\left\langle\beta^{2} \gamma^{2}\right\rangle\left\langle x^{2}\right\rangle\left\langle x^{\prime 2}\right\rangle-\langle\beta \gamma\rangle^{2}\left\langle x x^{\prime}\right\rangle^{2} .
$$

Considering the definition of relative energy spread $\sigma_{E}$,

$$
\sigma_{E}^{2}=\frac{\left\langle\beta^{2} \gamma^{2}\right\rangle-\langle\beta \gamma\rangle^{2}}{\langle\gamma\rangle^{2}}
$$

which, inserted in Eq. (2), and assuming relativistic electrons $(\beta=1)$, yields to 


$$
\varepsilon_{n}^{2}=\langle\gamma\rangle^{2}\left(\sigma_{E}^{2} \sigma_{x}^{2} \sigma_{x^{\prime}}^{2}+\varepsilon^{2}\right) .
$$

If the first term in the parentheses is negligible, we find the conventional definition of normalized emittance, as $\varepsilon_{n} \approx\langle\gamma\rangle \varepsilon$. For a conventional accelerator this might generally be the case, considering e.g. parameters of the SPARC photoinjector [18], at $5 \mathrm{MeV}$ the ratio between the first and the second term is $\approx 10^{-3}$ while at $150 \mathrm{MeV}$ it is $\approx 10^{-5}$. Using the parameters of our simulation we find that the first term of Eq. (4) at the plasma-vacuum interface is of the same order of magnitude as for conventional accelerators at low energies; however, due to the rapid increase of the bunch size, it becomes predominant compared to the second term. The use of simplified formulas for the measurement of the normalized emittance, such as performed in $[9,19,20]$, is therefore for laser-driven particle beams inappropriate. Considering the transverse beam size increase due to free diffraction, as indicated in [21], assuming as a starting condition a beam waist and a sufficiently long drift, we find that the bunch size becomes $\sigma_{x}(s) \approx \sigma_{x^{\prime}} s$ and Eq. (4) reads

$$
\varepsilon_{n}^{2}=\langle\gamma\rangle^{2}\left(s^{2} \sigma_{E}^{2} \sigma_{x^{\prime}}^{4}+\varepsilon^{2}\right) .
$$

Hence, the normalized emittance increases very rapidly and its rate can reach, with our parameters, a value of $1000 \mu \mathrm{m} / \mathrm{m}$. We have validated our calculation using the particle tracking code TSTEP, which is a derivative of PARMELA [22] and is considered a reference code in the conventional particle accelerator community. The inset of Fig. 2 compares the normalized emittance increase of an electron bunch in a drift as obtained using the analytical calculations above with that obtained using TSTEP, showing a good agreement.

The normalized emittance worsening [23] is due to the fact that the betatron frequency of a particle critically depends on its energy. During the drift, particles with

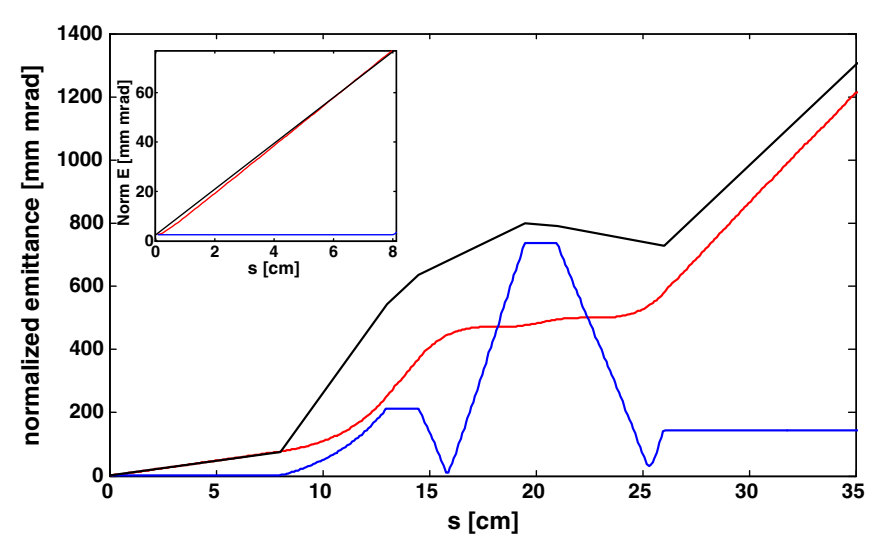

FIG. 2. Normalized transverse emittance along a transport line using magnetic quadrupoles: comparison between TSTEP simulations (red line), Eq. (4) (black line), and the $\gamma \varepsilon$ approximation (blue line). Inset: Zoom of the initial drift. different energies rotate with different velocities in the transverse phase space, widening the bunch trace space area; the resulting projected normalized emittance becomes a function both of drift length and energy spread. Additional emittance growth can be generated by the plasma down ramp length, as reported by $[10,20]$ : in our simulations the plasma down ramp length was $100 \mu \mathrm{m}$ long, much shorter than the betatron wavelength. A proper tailoring of the plasma down ramp would indeed produce an adiabatic defocusing of the bunch (this means that an increase of the transverse beam size would result in a decrease of the divergence keeping the emittance constant) and prevent a rapid normalized emittance dilution, as can be argued from Eq. (5) in our manuscript. However, this process would not affect the energy spread, so that the matching of the entire bunch to a transport line still remains very difficult and the normalized emittance still keeps a dependence from the drift length, although less pronounced.

Equation (4) can be also used to predict the normalized emittance behavior in presence of magnetic elements (e.g. quadrupoles). In Fig. 2 we show a comparison between Eq. (4), the expression $\langle\gamma\rangle \varepsilon$, and the normalized emittance obtained by TSTEP. The agreement between theory and simulation when using quadrupole devices is worse than for the case of a simple drift due to a different behavior of the beam when traveling within magnetic fields because of its high energy spread; however, Eq. (4) remains a valid analytical tool to determine the behavior of the normalized emittance of a beam after a laser-driven acceleration.

We stress that chromatic effects could be different for slice normalized emittance, which is the critical parameter for some applications such as free electron lasers (FELs) where small portions of the bunch (the slices) contribute almost independently to the emission of radiation. The length of the slice depends on the underlying physical processes; in FELs, it is approximately equal to the cooperation length, which is the length in which the radiation by the particles adds up coherently. In general, the energy spread of a bunch has two different contributions: the first one is the energy variation correlated with the longitudinal position of the particle in the bunch (e.g. due to wakefields or due to the variation of the rf voltage within the bunch), while the second one is the so-called uncorrelated energy spread which accounts for randomly distributed energy of the electrons inside the bunch. If we consider a thin slice, the energy correlation can usually be neglected compared to the uncorrelated energy spread. Therefore, if this last contribution is small, the beam slice would have the same behavior of a conventional beam. Figure 3 shows a comparison between normalized slice emittance at longitudinal distance $s=0$ and $s=1 \mathrm{~cm}$ (top plot) and the slice energy spread along the bunch (bottom plot). We can see from the bottom plot that the energy spread is relatively 


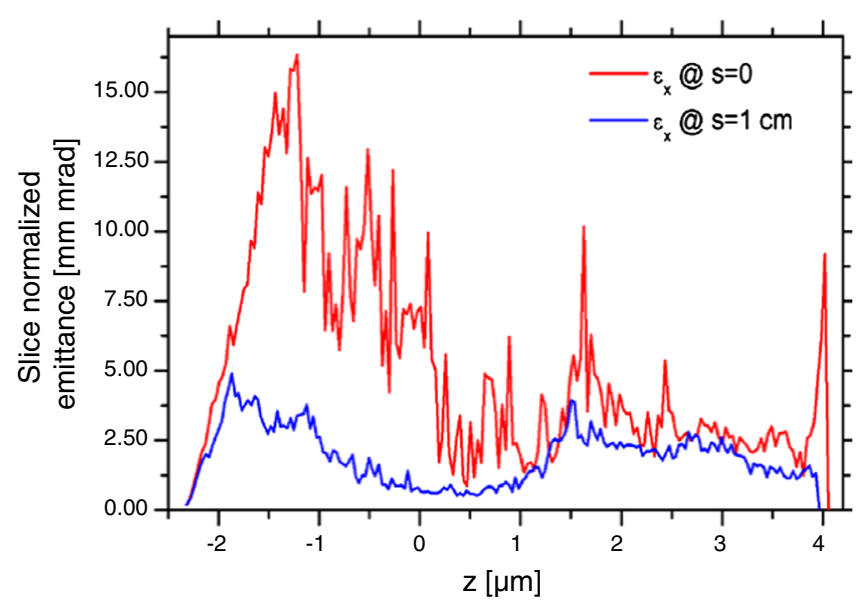

electron beams, conserving a decent percentage of its initial current and preserving a reasonable normalized emittance, requires therefore optical elements positioned close to the interaction point and with a focal length in the order of the bunch Twiss beta function. This is out of reach with the present-day magnet technology. We have shown that the beam worsening behavior is primarily due to the combined effect of a high energy spread and a high beam divergence. These parameters have therefore to be better controlled inside the plasma (e.g. with proper shaping of the plasma channel) if one plans to use this kind of source as an alternative to conventional particle accelerators.

\section{ACKNOWLEDGMENTS}

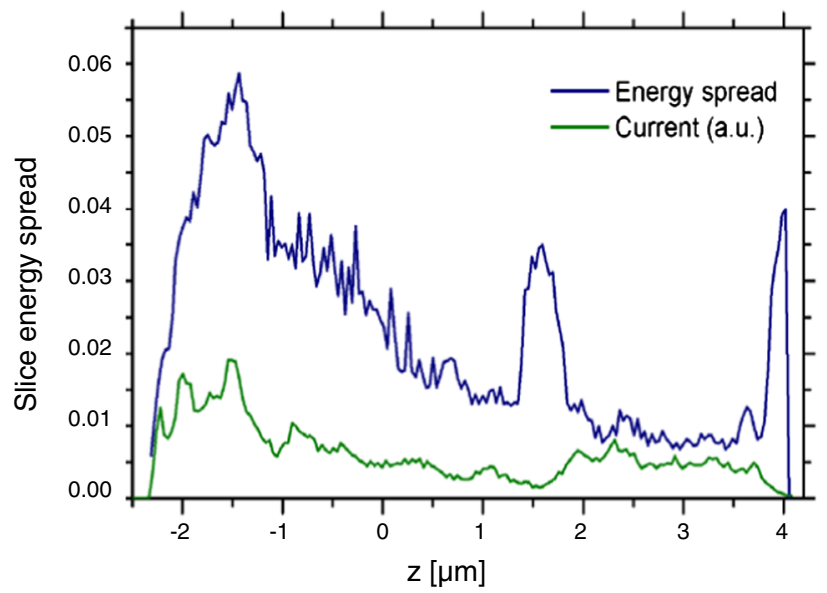

The authors acknowledge the fruitful discussions with the SPARC-Lab group and financial support from SPARXMIUR (Contract No. RBAP06AHF8_002), ELI-PP (FP7 Contract No. 212105), and CRISP (FP7 Contract No. 283745).

[1] The Future of Accelerator Physics, edited by T. Tajima (AIP, New York, 1996).

[2] B. Munroe, in Proceedings of IPAC2012, New Orleans, USA, TUPPR071 [http://www.jacow.org/].

[3] E. Esarey, C. Schroeder, and W. Leemans, Rev. Mod. Phys. 81, 1229 (2009).

FIG. 3. Comparison of slice normalized emittance at the interaction point and after a $1 \mathrm{~cm}$ drift space (top) and slice energy spread (bottom). The number of slices in the longitudinal plane is 200 .

low for the longitudinal position inside the bunch $(z)$ from 2 to $4 \mu \mathrm{m}$ and therefore, in the top plot, the slice emittance only differs slightly $(\leq 1 \%)$ in this region, compared to the region $z$ from -2 to $2 \mu \mathrm{m}$, where a strong worsening is visible. In fact, in different experiments in which laserdriven electrons were shaped using conventional accelerator elements in order to adapt them to different applications [24], the conventional accelerating elements were acting only on the part of the phase space that was adapted to the element and that could be considered as a conventional beam, making the source very lossy [25]. Therefore, more than shaping the beam, these elements are selecting a portion of the total charge, as can be seen in Ref. [24], in which the transported charge is reduced to $10 \%$ of the total one. This corresponds to the portion within the phase space in which the electron population has an acceptable energy spread to be transported with the conventional accelerator elements. In conclusion, we show that laser-driven electrons undergo a very strong, undesired normalized emittance worsening when leaving the interaction region, reaching quickly intolerable values. Capturing laser-driven

[4] C. A. Cechetti et al., Phys. Plasmas 16, 043102 (2009).

[5] C. G. R. Geddes, Cs. Toth, J. van Tilborg, E. Esarey, C. B. Schroeder, D. Bruhwiler, C. Nieter, J. Cary, and W.P. Leemans, Nature (London) 431, 535 (2004); S.P.D. Mangles et al., Nature (London) 431, 538 (2004); J. Faure, Y. Glinec, A. Pukhov, S. Kiselev, S. Gordienko, E. Lefebvre, J.-P. Rousseau, F. Burgy, and V. Malka, Nature (London) 431, 541 (2004).

[6] W.P. Leemans, B. Nagler, A. J. Gonsalves, Cs. Tóth, K. Nakamura, C. G. R. Geddes, E. Esarey, C. B. Schroeder, and S. M. Hooker, Nat. Phys. 2, 696 (2006).

[7] C. Rechatin, J. Faure, A. Ben-Ismail, J. Lim, R. Fitour, A. Specka, H. Videau, A. Tafzi, F. Burgy, and V. Malka, Phys. Rev. Lett. 102, 164801 (2009).

[8] A. J. Gonsalves et al., Nat. Phys. 7, 862 (2011).

[9] E. Brunetti et al., Phys. Rev. Lett. 105, 215007 (2010).

[10] C. M. S. Sears, A. Buck, K. Schmid, J. Mikhailova, F. Krausz, and L. Veisz, Phys. Rev. ST Accel. Beams 13, 092803 (2010).

[11] P. Antici et al., J. Appl. Phys. 112, 044902 (2012); P. Antici, C. Benedetti, D. Giacopello, M. Migliorati, A. Mostacci, and L. Palumbo, AIP Conf. Proc. 1228, 346 (2010).

[12] S. Gordienko and A. Pukhov, Phys. Plasmas 12, 043109 (2005).

[13] W. Lu, M. Tzoufras, C. Joshi, F. Tsung, W. Mori, J. Vieira, R. Fonseca, and L. Silva, Phys. Rev. ST Accel. Beams 10, 061301 (2007).

[14] C. Benedetti, A. Sgattoni, G. Turchetti, and P. Londrillo, IEEE Trans. Plasma Sci. 36, 1790 (2008); C. Benedetti, 
P. Londrillo, V. Petrillo, L. Serafini, A. Sgattoni, P. Tomassini, and G. Turchetti, Nucl. Instrum. Methods Phys. Res., Sect. A, 608, S94 (2009)

[15] C. Benedetti, AIP Conf. Proc. 1209, 11 (2010).

[16] http://ilil.ino.it/flame/index.html.

[17] http://www.lnf.infn.it/acceleratori/plasmonx.

[18] M. Ferrario et al., in Proceedings of FEL08, Gyeongju, Korea (2008), TUPPH048, p. 359 [http://www.jacow .org/].

[19] S. Fritzler, E. Lefebvre, V. Malka, F. Burgy, A. Dangor, K. Krushelnick, S. Mangles, Z. Najmudin, J.-P. Rousseau, and B. Walton, Phys. Rev. Lett. 92, 165006 (2004).
[20] S. Kneip et al., Phys. Rev. ST Accel. Beams 15, 021302 (2012).

[21] M. Reiser, Theory and Design of Charged Particle Beams (Wiley-VCH Verlag GmbH \& Co., Weinheim, (2004), p. 105, 2nd ed., Eq. (3.162).

[22] L. M. Young, Los Alamos National Laboratory Report No. LA-UR-96-1835.

[23] K. Floettmann, Phys. Rev. ST Accel. Beams 6, 034202 (2003).

[24] M. Fuchs et al., Nat. Phys. 5, 1404 (2009)

[25] Weingartner et al., Phys. Rev. ST Accel. Beams 14, 052801 (2011). 